EPJ manuscript No.

(will be inserted by the editor)

\title{
A new view of the electronic structure of the spin-Peierls compound $\alpha^{\prime}-\mathrm{NaV}_{2} \mathbf{O}_{5}$
}

P. Horsch and F. Mack

Max-Planck-Institut für Festkörperforschung, Heisenbergstr. 1, D-70569 Stuttgart (Germany)

January 28,1998

\begin{abstract}
The present understanding of the electronic and magnetic properties of $\alpha^{\prime}-\mathrm{NaV}_{2} \mathrm{O}_{5}$ is based on the hypothesis of strong charge disproportionation into $\mathrm{V}^{4+}$ and $\mathrm{V}^{5+}$, which is assumed to lead to a spin-1/2 Heisenberg chain system. A recent structure analysis shows, however, that the V-ions are in a mixed valence state and indistiguishable. We propose an explanation for the insulating state, which is not based on charge modulation, and show that strong correlations together with the Heitler-London character of the relevant intermediate states naturally lead to antiferromagnetic Heisenberg chains. The interchain coupling is weak and frustrated, and its effect on the uniform susceptibility is small.
\end{abstract}

Dedicated to J. Zittartz on the occasion of his 60th birthday

PACS. 75.50.Ee - 75.30.Et - 75.40.Cx - 75.40.Mg

One-dimensional spin-1/2 Heisenberg antiferromagnets are expected to undergo a structural phase transition into a dimerized phase at low temperature accompanied by the opening of a spin gap[1]. This spin-Peierls transition was first observed in organic systems [2], but has found considerable experimental attention after its recent discovery in $\mathrm{CuGeO}_{3}\left(T_{s p}=14 K\right)$ [3]. The $\alpha^{\prime}$-phase of $\mathrm{NaV}_{2} \mathrm{O}_{5}$ appears to be the second inorganic compound where a similar transition was observed with an even higher transition temperature $T_{s p}=34 \mathrm{~K}$ [幽. The size of the spin gap was determined by neutron scattering [5], Raman[6] and other experiments. The transition into the low-temperature dimerized structure was confirmed by X-ray scattering [0. NMR[7, thermal-expansion [8] yet the detailed deformation pattern is still unknown.

Based on an early structure determination for $\alpha^{\prime}-\mathrm{NaV}_{2} \mathrm{O}_{5}$ [9], the current picture for the origin of the one-dimensional magnetic properties rests on the assumption of charge discommensuration into $\mathrm{V}^{4+}$ and $\mathrm{V}^{5+}$ chains [4, [5, [1, 10, 11]. In Fig.1 the V1 and V2 chains in b-direction would corre- 
spond to these different charged vanadium chains. In this picture the $\mathrm{V}^{4+}$ chain would correspond to a spin- $1 / 2$ Heisenberg chain. A problem with this picture is, however, the missing physical argument for such a strong charge modulation.

In fact a recent new structural analysis of $\alpha^{\prime}-\mathrm{NaV}_{2} \mathrm{O}_{5}$ by von Schnering et al [12] clearly shows that all vanadiums are equivalent and in a mixed valent state. Furthermore the charge-ordered state at room temperature was found to be in conflict with Raman scattering experiments 13 . Since there is one d-electron per two $\mathrm{V}$ ions an explanation for the insulating properties must be found which does not rest on charge discommensuration.

The aim of this work is to show how in such an intermediate valence situation correlation effects can lead to a quasi-1D Heisenberg antiferromagnet. An important structural information 12, which is crucial for our analysis, are the V-O distances $d$ in the a-b plane (Fig.1): $d(\mathrm{~V} 1-$ $\mathrm{O} 3)=d(\mathrm{~V} 2-\mathrm{O} 3)=1.825 \AA, d(\mathrm{~V} 1-\mathrm{O} 2 \| \mathrm{b})=1.916 \AA$ and $d(\mathrm{~V} 1-$ $\mathrm{O} 2 \| \mathrm{a})=1.986 \AA$. This implies that the V1-O3-V2 bond is much shorter than the V1-O2-V1 bond in b-direction. This additional bonding effect can be attributed to the $d_{x y^{-}}$ electrons. From Fig. 1 we see that the $d_{x y}$-orbitals have a direct overlap in one direction, e.g. $\mathrm{V} 2-\mathrm{V}^{\prime}$, which is however quite small $t_{x y} \cong 0.3 \mathrm{eV}$ 14. Yet a $d_{x y}$-electron can also hop between V1 and V2 via a double exchange like process. In such a process first an electron hops from the occupied O3 $p_{y}$-orbital to $\mathrm{V} 2$ with an excitation energy $\Delta \epsilon_{y}=\epsilon\left(d_{x y}\right)-\epsilon\left(p_{y}\right)$, and in a second step the V1-electron annihilates the oxygen hole. The matrixelement for this

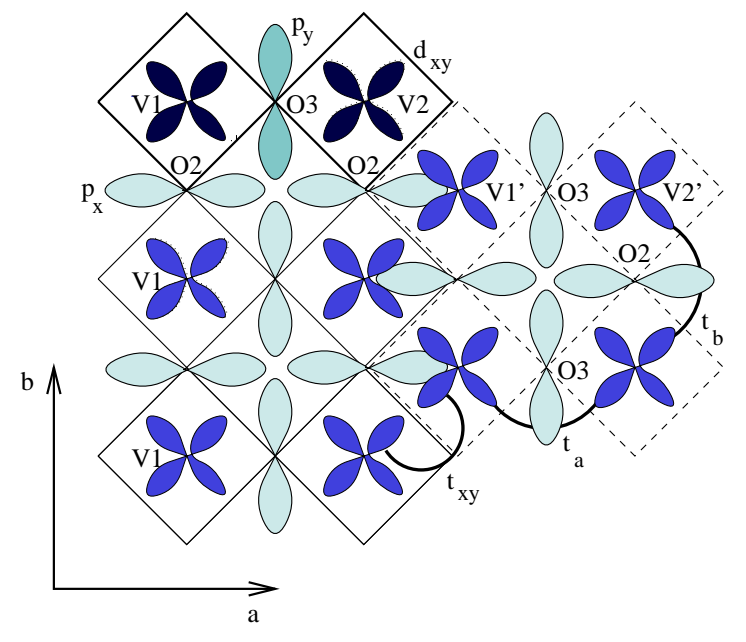

Fig. 1. Orbital structure of $\alpha^{\prime}-\mathrm{NaV}_{2} \mathrm{O}_{5}$ in the a-b plane. The lowest d-orbitals with $d_{x y}$-symmetry are occupied by one electron per two $\mathrm{V}$-atoms. The $\mathrm{O}$ p-orbitals at the corners of square pyramids (where only the basal plane is indicated) are occupied, with energies ranging from $-3 \mathrm{eV}$ down to $-7 \mathrm{eV}$. Solid (dashed) squares indicate the downward (upward) orientation of the pyramids (for structural details see Refs.[12] and [4]). The $d_{x y}$ orbitals have a direct overlap $t_{x y}$ along the V2-V1'$\mathrm{V} 2-\mathrm{V}^{\prime}$ zig-zag chain. The largest hopping matrix element $t_{a}$ is, however, via double exchange interaction involving an O3 $p_{y}$ orbital. The resulting bonding is manifested in the structure by the shortest planar V-O distance along the V1-O3-V2 bond.

process is $t_{a} \cong t_{p d}^{2} / \Delta \epsilon_{y}$. The matrixelement $t_{p d}$ depends on the vanadium-oxygen distance $d$ and can be estimated with the help of Harrison's 15] solid state table as $t_{p d}=$ $\eta_{p d \pi} \hbar^{2} r_{d}^{3 / 2} / m d^{7 / 2}$, where $\eta_{p d \pi}=1.36, \hbar^{2} / m=7.62 \mathrm{eV} \AA^{2}$, and $r_{d}=0.98 \AA$ for vanadium. This gives $t_{p d} \cong 1.2 \mathrm{eV}$. The $p$ - $d$ excitation energies are determined from a LMTObandstructure calculation [14], which yields $\Delta \epsilon_{y} \cong 4 \mathrm{eV}$ 
and $\Delta \epsilon_{x} \cong 6.5 \mathrm{eV}$, respectively. Hence $t_{a} \cong 0.35 \mathrm{eV}$, while the corresponding matrixelement $t_{b} \cong 0.15 \mathrm{eV}$ is considerably smaller because of the larger oxygen-vanadium distance and the larger $\Delta \epsilon_{x}$. We note that the larger value for $t_{a}$ is consistent with the stronger bonding of the V1O3-V2 bond.

The Hamiltonian for the $d_{x y}$-electrons may be written in terms of creation and density operators $d_{\mathbf{i} \alpha \sigma}^{\dagger}$ and $n_{\mathbf{i} \alpha \sigma}=$ $d_{\mathbf{i} \alpha \sigma}^{\dagger} d_{\mathbf{i} \alpha \sigma}$, respectively, in the form

$$
\begin{aligned}
H_{d}= & -\sum_{\mathbf{i} \sigma} t_{a}\left(d_{\mathbf{i} 1 \sigma}^{\dagger} d_{\mathbf{i} 2 \sigma}+H . c .\right)+U_{d} \sum_{\mathbf{i} \alpha} n_{\mathbf{i} \alpha \uparrow} n_{\mathbf{i} \alpha \downarrow} \\
& -\sum_{\langle\mathbf{i}\rangle \alpha \beta \sigma} t_{\mathbf{i j}}\left(d_{\mathbf{i} \alpha \sigma}^{\dagger} d_{\mathbf{j} \beta \sigma}+H . c .\right)
\end{aligned}
$$

where we have introduced a cell structure. Here a cell contains two vanadium atoms V1 and V2, i.e. $\alpha=1$ and 2 , and is labeled by a cell index $\mathbf{i}=\left(i_{a}, i_{b}\right)$. The cellHamiltonian consists of a kinetic energy term $t_{a}$ and the local interaction $U_{d} \cong 4 \mathrm{eV}$ 16]. In $H_{d}$ we droped a shift of the single particle levels which is only relevant for total energy considerations 17 . The hopping between cells is defined by the last term, where

$$
t_{\mathrm{ij}}=\left\{\begin{array}{c}
t_{b} \text { for } \alpha=\beta, j_{a}=i_{a}, j_{b}=i_{b} \pm 1 \\
t_{x y} \text { for } \alpha \neq \beta, j_{a}=i_{a} \pm 1, j_{b}=i_{b} \pm \frac{1}{2}
\end{array}\right.
$$

Since the ratios between $U_{d}$ and the various hopping matrix elements are quite large, we are confronted with a strong correlation problem.

In the following we shall use a cell-perturbation method 18 based on the above cell structure, where each V1-V2 cell contains in the average a single $d$-electron. The advantage of the cell decomposition is the capability to treat the local correlations exactly. The complete set of states

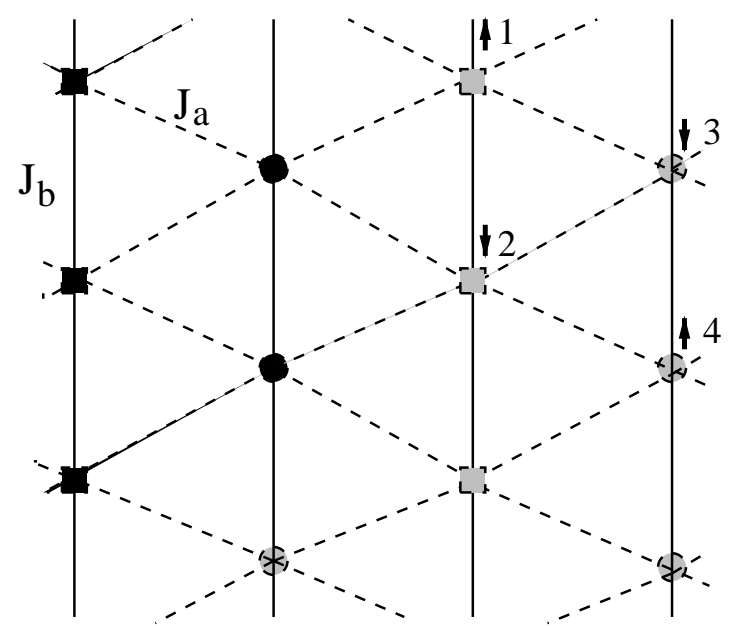

Fig. 2. Geometric structure of the effective spin model. A site corresponds to a V1-O3-V2 cell with a single $d$-electron. Dark symbols indicate the part of the structure shown in Fig. 1. The largest antiferromagnetic interaction is $J_{b}$, while the frustrated interaction $J_{a}$ between neighboring b-chains is small. The one-dimensional magnetic structure is further enhanced by the topology of the lattice. The antiferromagnetic interaction $J_{a}$ of spin 3 with spins 1 and 2 on the neighbor chain is frustrated.

is then labeled by the quantum numbers of the cells, i.e. including the number of electrons within a cell. In the one electron sector the cell states are simply bonding and antibonding states at energies $\pm t_{a}$ and corresponding operators $b_{\mathbf{i} \sigma}^{\dagger}\left(a_{\mathbf{i} \sigma}^{\dagger}\right)=\frac{1}{\sqrt{2}}\left(d_{\mathbf{i} 1 \sigma}^{\dagger} \pm d_{\mathbf{i} 1 \sigma}^{\dagger}\right)$, respectively. The low energy configurations $b_{\mathbf{i} \sigma}^{\dagger} b_{\mathbf{j} \sigma^{\prime}}^{\dagger}$ of two electrons in neighbor cells $\mathbf{i}$ and $\mathbf{j}$ are coupled in second order due to the hopping $t_{\mathbf{i j}}$. The intermediate states have two electrons in one cell. The low-energy singlet and triplet states have excitation energies $\Delta E_{s}=2 t_{a}-\frac{1}{2}\left(\sqrt{U_{d}^{2}+16 t_{a}^{2}}-U_{d}\right)$ and $\Delta E_{t}=2 t_{a}$, respectively. Other singlet states are at much higher en- 
ergy $E \geq U_{d}+2 t_{a}$. The Heitler-London singlet-triplet splitting of the low-energy intermediate states turns out to be crucial for the anisotropic nature of the magnetic properties.

The coupling of the $b_{\mathbf{i} \sigma}^{\dagger} b_{\mathbf{j} \sigma^{\prime}}^{\dagger}$ configurations may be expressed in compact form by the spin-1/2 Hamiltonian

$$
H=\sum_{\langle\mathbf{i} \mathbf{j}\rangle}\left[J_{\mathbf{i j}}^{s}\left(\mathbf{S}_{\mathbf{i}} \mathbf{S}_{\mathbf{j}}-\frac{1}{4} n_{\mathbf{i}} n_{\mathbf{j}}\right)-J_{\mathbf{i j}}^{t}\left(\mathbf{S}_{\mathbf{i}} \mathbf{S}_{\mathbf{j}}+\frac{3}{4} n_{\mathbf{i}} n_{\mathbf{j}}\right)\right]
$$

where $\mathbf{S}_{\mathbf{i}}=\frac{1}{2} b_{\mathbf{i}, \sigma}^{\dagger} \tau_{\sigma \sigma^{\prime}} b_{\mathbf{i}, \sigma^{\prime}}$ defines the spin of bonding electrons in terms of the vector of Pauli spin matrices $\tau$ and $n_{\mathbf{i}}=\sum_{\sigma} b_{\mathbf{i}, \sigma}^{\dagger} b_{\mathbf{i}, \sigma}$ their density. Here $J_{\mathbf{i j}}^{s}$ and $J_{\mathbf{i j}}^{t}$ denote the coupling of neighboring cells in the singlet- and triplet channel, respectively. The relative size of the exchange integrals is strongly influenced by the geometrical structure (Fig. 1 and 2). The coupling of cells in a-direction, i.e. via $t_{x y}$, yields $J_{a}^{s}=2\left(t_{x y} / 2\right)^{2} / \Delta E_{s}$ and $J_{a}^{t}=2\left(t_{x y} / 2\right)^{2} / \Delta E_{t}$. They differ only because of the singlet-triplet splitting $E_{s-t}=\frac{1}{2}\left(\sqrt{U_{d}^{2}+16 t_{a}^{2}}-U_{d}\right)$. The coupling of cells in bdirection is $J_{b}^{s}=2 t_{b}^{2} / \Delta E_{s}$ and $J_{b}^{t}=0$, where the latter exchange integral vanishes due to symmetry. This has the effect that the total exchange constants in $H=\sum J_{\mathrm{ij}} \mathbf{S}_{\mathrm{i}} \mathbf{S}_{\mathbf{j}}$ almost cancel along a-direction, i.e. $J_{a}=J_{a}^{s}-J_{a}^{t}$, whereas along the 'b-chains' $J_{b}=J_{b}^{s}$ there is no such reduction. These estimates are valid if the ratios $\frac{2 t_{a}}{U}$ and $\frac{t^{\prime}}{2 t_{a}}$, with $t^{\prime}=\left\{t_{b}, t_{x y} / 2\right\}$, are small compared to 1 . Numerical estimates for these exchange integrals based on degenerate perturbation theory are shown in Fig. 3 as function of the vanadium-oxygen (V1-O3) hybridization $t_{p d}$. For the estimated value $t_{p d} \simeq 1.2 \mathrm{eV}$ one finds $J_{b} \simeq 75 \mathrm{meV}$ and $J_{a} \simeq 13 \mathrm{meV}$.

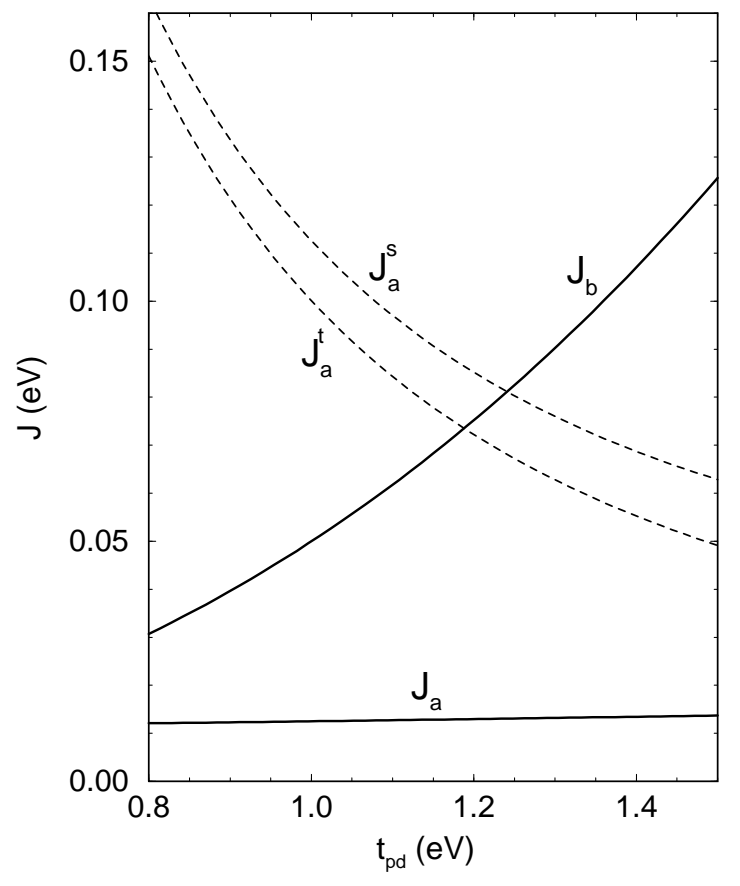

Fig. 3. AF-exchange coupling between d-electrons in nearest neighbor V1-O3-V2 bonds as function of $t_{p d}$ : (a) $J_{b}$ along b-direction (solid line), (b) the small coupling $J_{a}=J_{a}^{s}-J_{a}^{t}$ between different b-chains mediated by $t_{x y} \sim 0.3 \mathrm{eV}$ results from the cancellation of the contributions $J_{a}^{s}$ and $J_{a}^{t}$ from the Heitler-London split singlet and triplet intermediate states (dashed lines).

Experimental estimates for the exchange constants are usually obtained from the position of the maximum of the uniform susceptibility $\chi(T)$. In the following we study the effect of the interchain coupling $J_{a}$ and the thereby introduced frustration of the spin-1/2 model (3) using a finite temperature diagonalization technique 19 . The geometrical structure of the lattice (Fig. 2) is similar to the resonating valence bond systems studied by Anderson and Fazekas [20]. Results for a two-leg ladder ('railroadtrestle') with periodic boundary conditions along a- and b- 
P. Horsch and F. Mack: A new view of the electronic structure of the spin-Peierls compound $\alpha^{\prime}-\mathrm{NaV}_{2} \mathrm{O}_{5}$

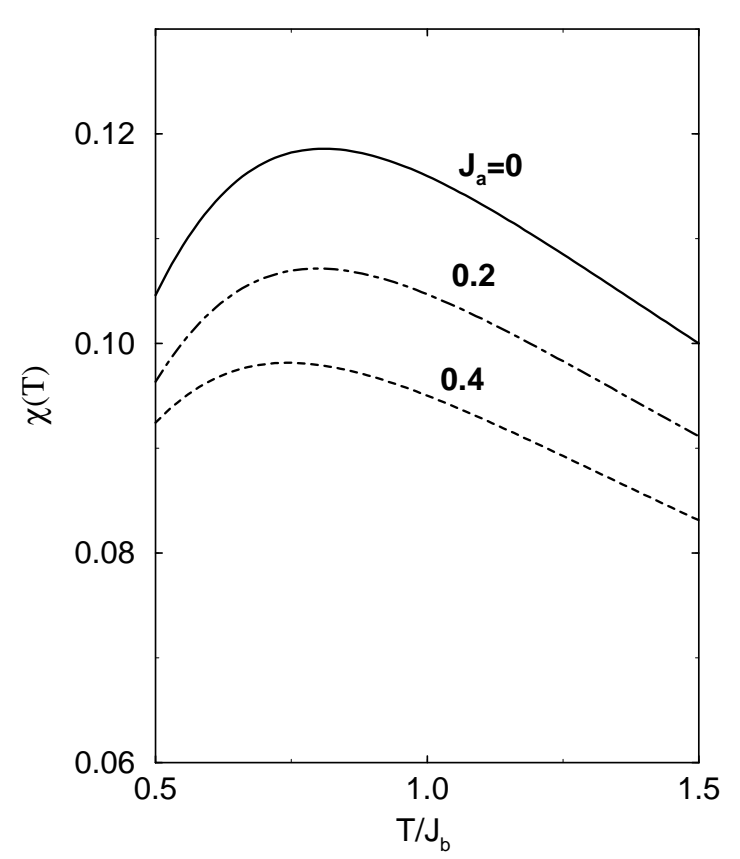

Fig. 4. Uniform susceptibility $\chi(T)$ for a two-leg ladder $(2 \times 12)$ with the structure given in Fig. 2 for different interchain coupling strength $J_{a} / J_{b}=0,0.2$ and 0.4 and periodic boundary conditions.

direction and different interchain coupling strength $J_{a} / J_{b}$ are shown in Fig. 4 and 5. Since the interchain coupling is frustrated the change of $\chi(T)$ is relatively small. The maximum of $\chi(T)$ is at the temperature $T_{\chi}^{\max }=a_{\chi} J_{b}$, where $a_{\chi}=0.8$ for a $2 \times 12$ system in the absence of the interchain coupling, i.e. $J_{a} / J_{b}=0$. The exact result for the thermodynamic limit recently obtained by Eggert et al. 21 using the thermal Bethe ansatz is $a_{\chi}=0.6$. Interchain coupling leads to a small shift of the maximum by about $2 \%$ to lower temperatures for the value $J_{a} / J_{b} \sim 0.2$ estimated above (Fig. 4).

We note that lattice fluctuations are expected to lead to a further weakening of antiferromagnetism and an additional shift of the maximum of $\chi(T)$ to lower $T$. A re-

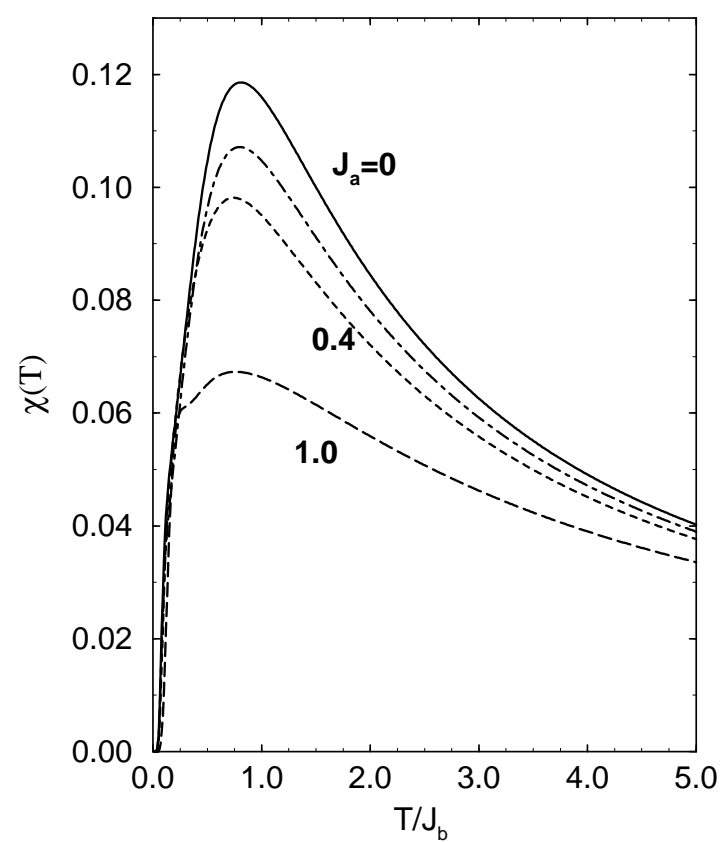

Fig. 5. Uniform susceptibility $\chi(T)$ for a $2 \times 12$ two-leg ladder for different interchain coupling strength $J_{a} / J_{b}=0,0.2,0.4$ and 1.0 on a large temperature scale. The rapid drop below $T / J_{b} \leq 0.2$ is a finite size effect.

cent study of this effect by Sandvik et al.22] shows that this may lead to a reduction of $T_{\chi}^{\max }$ by $15 \%$ for a onedimensional Heisenberg chain. Combining these two effects one arrives at the estimate $a_{\chi}^{\text {tot }} \sim 0.50$. From the susceptibility measurements [4, 10] for $\alpha^{\prime}-\mathrm{NaV}_{2} \mathrm{O}_{5}$ one finds $T_{\chi}^{\max } \sim 350 \mathrm{~K}$, which leads to the estimate $J_{b}^{\text {exp }} \sim 700 \mathrm{~K}$. This is in reasonable agreement with the theoretical value $J_{b} \sim 75 \mathrm{meV}$ derived in this work. In view of the simplicity of the model and the rough estimates of the parameters this is quite satisfactory.

We briefly comment on the band picture, i.e. ignoring the effect of electron correlations. Since double-exchange is not contained in this scheme, the only large hopping matrix element is $t_{x y}$. This leads to two degenerate one- 
dimensional $d_{x y}$-bands from the zig-zag chains $\mathrm{V} 2-\mathrm{V}^{\prime}$ $\mathrm{V} 2-\mathrm{V} 1{ }^{\prime}$ and $\mathrm{V} 2^{\prime}-\mathrm{V} 1-\mathrm{V} 2$ '-V1 (Fig. 1), respectively, since there are two zig-zag b-chains per unit cell. Each band is quarter-filled and one may expect a usual Peierls transition to occur due to an appropriate dimerization of the structure in b-direction. Although this could explain the insulating properties at room temperature, such an explanation is obviously incorrect, since it would rule out a spinPeierls transition at low temperature. Of course the large ratio $U_{d} / t_{x y}$ already excludes the straightforward application of the band picture. Nevertheless for sufficiently large $t_{x y}$ one expects that the correlated band picture applies. Estimates of the ground state energies for the localized regime and the band picture suggest that this is the case for $t_{x y}>1.5 t_{a}$.

The bands obtained by a real bandstructure calculation deviate from this idealistic picture due to small interchain couplings, which is also reflected in shorter V1-O3V2 bonds 14. The full bandstructure calculation predicts a metallic state.

We remark that photoemission experiments in combination with XAS would provide a sensitive test of the present picture, since the lowest unoccupied states are the singlet and triplet states, while higher lying 'two-particle' states are split by $U_{d}$. Optical conductivity measurements on the other hand should show a relatively small 'single particle' gap of order $2 t_{a}$.

In summary we have shown that $\alpha^{\prime}-\mathrm{NaV}_{2} \mathrm{O}_{5}$ is an insulator due to strong correlations, i.e. large $U_{d}$. However since the single electron orbitals have bonding character, i.e. involving two $\mathrm{V}$-atoms, the magnetic structure is induced via 2-electron molecular singlet and triplet states which are Heitler-London split. Due to the almost perfect cancellation of the triplet- and singlet-interactions in adirection the spin system is essentially one-dimensional. We stress that doubly occupied states with energy $\sim U_{d}$, which usually contribute to the magnetic interaction in Mott-Hubbard insulators, have little influence since they are at much higher energy. Therefore we suggest the name Heitler-London insulator as a more precise characterization for such systems.

We acknowledge stimulating discussions with J. van den Brink, Yu. Grin, A. M. Oles and H.-G. von Schnering.

\section{References}

1. E. Pytte, Phys. Rev. B 10, 4637 (1974); M. C. Cross and D. S. Fisher, Phys. Rev. B 19, 402 (1979).

2. J. W. Bray et al., Phys. Rev. Lett. 35, 744 (1975);

3. M. Hase, I. Terasaki and K. Uchinokura, Phys. Rev. Lett. 70, 3651 (1993).

4. M. Isobe and Y. Ueda, J. Phys. Soc. Jpn. 65, 1178 (1996). 5. Y. Fujii, H. Nakao, T. Yoshihama, M. Nishi, K. Nakajima, K. Kakurai, M. Isobe. Y. Ueda, and H. Sawa, J. Phys. Soc. Jpn. 66, 326 (1997).

6. M. Weiden, R. Hauptmann, C. Geibel, F. Steglich, M. Fischer, P. Lemmens, and G. Güntherodt, Z. Phys. B 103, 1 (1997).

7. T. Ohama, M. Isobe, H. Yasuoka, and Y. Ueda, J. Phys. Soc. Jpn. 66, 545 (1997).

8. M. Köppen, D. Pankert, R. Hauptmann, M. Lang, M. Weiden, C. Geibel, and F. Steglich, cond-mat/9710183. 
P. Horsch and F. Mack: A new view of the electronic structure of the spin-Peierls compound $\alpha^{\prime}-\mathrm{NaV}_{2} \mathrm{O}_{5}$

9. A. Carpy and J. Galy, Acta Crystallgr., Sect B 31, 1481

(1975).

10. F. Mila, P. Millet, and J. Bonvoisin, Phys. Rev. B 54, 11925 (1996).

11. D. Augier, D. Poilblanc, S. Haas, A. Delia, and E. Dagotto, Phys. Rev. B 97, R5732 (1997).

12. H.-G. von Schnering, Yu. Grin, M. Kaupp, M. Somer, R. Kremer, O. Jepsen, T. Chatterji, and M. Weiden, to be published.

13. M. Fischer, G. Els, P. Lemmens, G. Güntherodt, A. S. Mishenko, M. Weiden, R. Hauptmann, C. Geibel, and F. Steglich, to be published.

14. Yu. Grin (private communication).

15. W. A. Harrison, Electronic Structure and Properties of Solids (Freeman, San Francisco, 1980).

16. H. F. Pen, PhD-thesis Groningen (1997), and private communication.

17. Covalency effects lead to diagonal shifts of $-4 t_{a},-2 t_{a}-t_{a}^{U}$ and $-2 t_{a}^{U}$ in the $n_{d}=0,1$ and 2 sectors, respectively, where $t_{a}^{U} \sim t_{p d}^{2} /\left(e_{d}-e_{p}+U_{d}\right)$. Although these shifts are important for ground state energies, they do not affect the excitation energies considered here.

18. J. H. Jefferson, H. Eskes, and L .F. Feiner, Phys. Rev. 45, 7959, (1992).

19. J. Jaklič and P. Prelovšek, Phys. Rev. B 49, 5065 (1994).

20. P. W. Anderson, Mat. Res. Bull. 8, 153 (1973), P. Fazekas and P. W. Anderson, Phil. Mag. 30, 423 (1974).

21. S. Eggert, I. Affleck, and M. Takahashi, Phys. Rev. Lett. 73, 332 (1994).

22. A. W. Sandvik, R. R. P. Singh, and D. K. Campbell, Phys.

Rev. B 56, 14510 (1997) . 